\title{
Lifestyle correlates of dietary patterns among young adults: evidence from an Australian birth cohort
}

\author{
Tolassa W Ushula ${ }^{1,2, *}$, Petra H Lahmann ${ }^{1}$, Abdullah Mamun ${ }^{3,4,5}$, William YS Wang ${ }^{6}$, \\ Gail $M$ Williams ${ }^{1}$ and Jake $M$ Najman ${ }^{1}$ \\ ${ }^{1}$ School of Public Health, Faculty of Medicine, The University of Queensland, Brisbane, Australia: ${ }^{2}$ Nutrition and \\ Dietetics Department, Faculty of Public Health, Jimma University, Jimma, Ethiopia: ${ }^{3}$ Institute for Social Science \\ Research, The University of Queensland, Brisbane, Australia: ${ }^{4}$ The Australian Research Council (ARC) Centre of \\ Excellence for Children and Families over the Life Course, The University of Queensland, Brisbane, Australia: ${ }^{5}$ The \\ Queensland Alliance for Environmental Health Sciences, The University of Queensland, Brisbane, Australia: ${ }^{6}$ Faculty of \\ Medicine, The University of Queensland, Princess Alexandra Hospital, Brisbane, Australia
}

Submitted 2 December 2020: Final revision received 25 August 2021: Accepted 2 September 2021: First published online 6 September 2021

\begin{abstract}
Objective: Previous studies of sociodemographic and lifestyle correlates of dietary patterns among young adults have primarily focused on physical activity and smoking, with inconclusive results. This study aims to examine the associations between a broader range of lifestyles of young adults and their patterns of food consumption. Design: Cross-sectional.

Setting: Brisbane, Australia.

Participants: The data set are from a long running birth cohort study which commenced in 1981. Details of dietary intake and sociodemographic and lifestyle factors were from the 21-year follow-up of the Mater-University of Queensland Study of Pregnancy (MUSP) birth cohort. The effective cohort ( $n$ 2665, 57\% women) is of young adult offspring. Usual dietary intake was assessed using a Food Frequency Questionnaire (FFQ). Data on sociodemographic and lifestyle variables were obtained from self-reports.

Results: Western and prudent dietary patterns were identified for the combined cohort of women and men using principal components analysis. Multivariable linear regression models were used to examine the associations between lifestyle variables and dietary patterns adjusting for potential confounders. Results from multivariable adjusted models showed that physical activity, watching TV and smoking were strongly associated with each dietary pattern; alcohol consumption and BMI showed weaker associations $(P<0.05$ for all).

Conclusions: Our study describes a clustering of unhealthy lifestyles in young adults. Young adults with unhealthy lifestyles less often adhere to a healthy prudent dietary pattern and more often an unhealthy Western pattern. Dietary preferences are enmeshed in a lifestyle matrix which includes physical activity, sedentary activity, smoking and alcohol consumption of young adults.
\end{abstract}



Dietary and other modifiable lifestyle habits including cigarette smoking, alcohol consumption, physical inactivity, snacking, skipping breakfast and higher body weight have been causally linked to long-term adverse health outcomes including morbidity and mortality ${ }^{(1)}$. There is little known about the extent to which dietary patterns are a more general component of lifestyle during young adulthood. Young adulthood is characterised by extensive lifestyle changes and explorations ${ }^{(2)}$. While the underlying contributing factors to the development and clustering of unhealthy lifestyles are the subject of a body of research, socio-cultural and environmental factors are believed to play important roles in these processes ${ }^{(3,4)}$. Unhealthy lifestyles including poor dietary habits, smoking, alcohol consumption and physical inactivity as well as screen-based/ sedentary activity can be observed during young adulthood $^{(5-8)}$. These lifestyles, particularly poor dietary habits, sedentary activity and smoking, develop early in the life course and are likely to track through adolescence and into young adulthood ${ }^{(9-12)}$. The multifaceted lifestyles observed

***Corresponding author: Email t.wakayoushula@uq.net.au, tolassawakayo@gmail.com

(C) The Author(s), 2021. Published by Cambridge University Press on behalf of The Nutrition Society. This is an Open Access article, distributed under the terms of the Creative Commons Attribution licence (https://creativecommons.org/licenses/by/4.0/), which permits unrestricted re-use, distribution, and reproduction in any medium, provided the original work is properly cited. 
during young adulthood may be interrelated and may be associated with poor food choices ${ }^{(13-17)}$ and bad eating habits: skipping meals; eating away from home; and include a reliance on fast foods and energy dense snacks ${ }^{(6,7)}$. It is plausible that dietary habit is part of a broader aspect of lifestyle and that lifestyle interventions may be necessary to facilitate better food choices and better eating habits. In this study, we report findings from the Mater University of Queensland Study of Pregnancy (MUSP) birth cohort to examine the associations between the dietary patterns of respondents and four key lifestyle factors, specifically smoking, alcohol consumption, physical activity and screen-based/sedentary activity in young adult offspring at the 21-year follow-up.

A few studies have specifically focused on lifestyle behaviours and their associations with dietary patterns among young adults ${ }^{(18)}$. There are some studies which examine sociodemographic and lifestyle variables influencing dietary pattern of young adults. We identified seven such studies conducted in the USA, Brazil, Denmark, UK and Canada including men and women aged between 18 and 39 years $^{(19-25)}$. These studies have largely focused on smoking ${ }^{(18-20,23)}$ and physical activity ${ }^{(19,23,24)}$ as lifestyle variables, with conflicting results. For example, in some studies less healthy eating patterns have been associated with lower level of physical activity ${ }^{(19,23)}$ and smoking $^{(19,20,23)}$ in young adults. A high level of physical activity has been associated with increases in prudent dietary pattern scores over time among young women in the $\mathrm{UK}^{(24)}$. By contrast, smoking has shown no association with any of the identified dietary patterns in this later study of young women in the $\mathrm{UK}^{(24)}$ and in another study of men and women in the USA ${ }^{(18)}$. A few studies that have considered alcohol consumption ${ }^{(19)}$ and sedentary activity ${ }^{(18,24)}$ have not observed association with the dietary patterns they examined.

There is also limited evidence regarding BMI as a possible lifestyle correlate of dietary patterns, particularly during the period of emerging adulthood when weight gain is likely high ${ }^{(26,27)}$. While BMI appears unrelated to changes in dietary patterns over 2 years among young women in the $\mathrm{UK}^{(24)}$, other studies that have adjusted for a confounding effect of BMI in predicting dietary patterns have not reported estimates for $\mathrm{BMI}^{(20,22,23)}$.

In general, previous studies have focused largely on physical activity and smoking and overlooked other lifestyle factors such as alcohol consumption, sedentary activity and body weight in relation to patterns of eating among young adults. Moreover, there is a mounting body of evidence showing sociodemographic characteristics including older age, female gender and both higher income and education are positively associated with the healthier food choices ${ }^{(28,29)}$ as well as healthier dietary patterns ${ }^{(20-23)}$ in young adults. We hypothesise that both lifestyles and dietary patterns are, in part, a consequence of the social contexts in which a person is reared. In this study, we examine whether dietary patterns are correlated with a range of lifestyles in young adults adjusting for potentially confounding effects of such social contexts including levels of income and education. Specifically, we examine physical activity, sedentary activity, cigarette smoking, alcohol consumption and BMI and their associations with dietary patterns in young Australian women and men at a mean age of 21 years using a large populationbased data set.

\section{Methods}

\section{Participants and design}

The present study uses offspring data from the MUSP, a prospective birth cohort study of mother-offspring pairs in Brisbane, Australia. Between 1981 and 1983, all women ( $n$ 8556) who presented for their first antenatal visit at the Mater Hospital in Brisbane were asked to participate in the study; $98 \%$ of those attending the booking in clinic were enrolled in the study and 6753 women who gave birth to 7223 children that were not adopted out before leaving the hospital, constitute the MUSP birth cohort. At the 14- and 21-years follow-ups, the offspring completed health, social and lifestyle questionnaires. Details of the MUSP birth cohort including recruitment of participants and measurements taken at each follow-up visit have been previously published ${ }^{(30)}$.

This current study examines data from the 21-year follow-up (2001-2003) of the offspring. A total of 3805 $(52.7 \%)$ offspring, young adults from this point forward, responded to a core questionnaire and 3654 (1731 men; 1923 women) completed a food frequency questionnaire $(\mathrm{FFQ})^{(30,31)}$. A core questionnaire addressed the following topics: sociodemographic characteristics; lifestyles; general health and well-being; and other topics such as psychosocial and mental health of respondents. Exclusions (total $n$ 894) were based on prior work with this cohort ${ }^{(31)}$. We excluded 429 young adults with incomplete FFQ information, such as a missing or blank FFQ page or whole section, or which had a lack of information on alcohol consumption. Another 465 young adults were excluded because of implausible energy intake reports that are defined as $<500$ or $\geq 3500 \mathrm{kcal} / \mathrm{d}$ for female participants and $<800$ or $\geq 4000 \mathrm{kcal} / \mathrm{d}$ for male participants ${ }^{(31)}$. We only included participants with $<40 \%$ missing FFQ items (blanks), leaving an analytical cohort of 2760 young adults with useable dietary data, out of which 2665 (1135 men, 1530 women) had complete data for each variable included in the current analyses (see online Supplemental Fig. 1).

\section{Assessment of lifestyles}

In this study, we consider five measures of lifestyle, namely smoking status, alcohol consumption, physical activity and 
sedentary/screen-based activity as well as BMI based on availability of the data and previous literature evaluating possible associations of some of these variables with dietary patterns among young adults ${ }^{(18-25)}$. Information on each lifestyle measure comprised a self-report provided by young adults.

Smoking status was measured as the number of cigarettes per day in three categories; non-smoker, 1-19 cigarettes (light smokers) or $\geq 20$ cigarettes (heavy smokers). Alcohol consumption was measured based on estimates of the mean number of standard drinks consumed/d; abstainer (0), light consumer (0.1-0.5), moderate consumer $(0 \cdot 51-1)$, heavy consumer (1.01-3.4) or very heavy consumer $(\geq 3.5)$. This latter variable was aggregated into four categories as abstainers, and light, moderate and heavy consumers, for further analysis.

Further, participants were asked about their physical activity using questions from the Active and Inactive Australians Questionnaire (AIAQ) ${ }^{(32)}$. The AIAQ questionnaire includes questions about vigorous exercise, less vigorous exercise and walking along with average weekly sessions ('never', '1-2 times' and ' 3 or more times') of engagement in each type of activity during the past 6 months $^{(32)}$. Exercise was considered vigorous when it made a participant breath harder or puff and pant including such activities as swimming, tennis, netball, athletics and running and less vigorous when it was for recreation purpose including such activities as bike riding and dancing. We quantified categories of sessions and summed up these values to obtain a weekly average number of sessions that a participant was engaged in any type of physical activity ${ }^{(33)}$. Participants were then grouped as physically inactive ( 0 sessions), insufficient ( $1-5$ sessions) and sufficient ( $\geq 5$ sessions) physical activity for health based on Active Australia Survey guideline ${ }^{(34)}$.

In addition, participants were assessed for sedentary/ screen-based activity, defined by time spent watching television (TV), using daily number of hours they spent watching TV (weekend days and weekdays) separately with the following response options: 'never watched' and watched for '< $<$ h', ' $1-3$ h', ' $3-5$ h', '5-7 h' and ' 7 h or more'. We quantified categories of each response to estimate the average daily time spent watching $\mathrm{TV}^{(33)}$. First, we calculated the average values of time spent on weekdays and on weekend days and multiplied by five and two, respectively. We summed up these values and divided them by seven to obtain daily average time spent watching TV for each participant. Participants were then grouped into three categories as $<2 \mathrm{~h} / \mathrm{d}$ (bottom $25 \%$ ), $2-4 \mathrm{~h} / \mathrm{d}$ (middle $50 \%$ ) or $>4 \mathrm{~h} / \mathrm{d}$ (top $25 \%$ ) duration of time spent watching TV based on the relative distribution of daily average time spent for this purpose.

BMI $\left(\mathrm{kg} / \mathrm{m}^{2}\right)$ was calculated separately from measured ( $n$ 1967) and self-reported ( $n$ 2681) weight $(\mathrm{kg})$ and height $(\mathrm{m})$ of participants and categorised into three groups based on WHO criteria; normal weight $\left(<25 \mathrm{~kg} / \mathrm{m}^{2}\right)$, overweight $\left(25-30 \mathrm{~kg} / \mathrm{m}^{2}\right)$ or obese $\left(>30 \mathrm{~kg} / \mathrm{m}^{2}\right)^{(35)}$. We found a strong correlation $(r=0.98$; $P<0.0001)$ between BMI calculated from measured and self-reported data. This finding is similar to a report from a nationally representative sample of adult Australians ${ }^{(36)}$. Hence, we used BMI calculated from self-reported data to maintain a larger cohort (Table 1). Arguably, people may choose to consume healthy foods to maintain their normal body weight or they may modify their food choices when they become obese in order to reduce their body weight. Thus, we considered BMI as one of the modifiable lifestyle variables that could influence dietary intakes of persons $^{(37,38)}$.

\section{Assessment of covariates}

A number of sociodemographic characteristics including living arrangements have been associated with dietary patterns $^{(20-23,31,39)}$, whereas dietary supplement use has been associated with the adoption of other healthy habits such as a healthier diet, better physical activity, avoiding smoking and alcohol consumption and healthy body weight ${ }^{(40)}$. We used self-reports of young adults to obtain information on sociodemographic measures. These measures include age (in years), gender, level of education (incomplete secondary school, secondary school, CollegeTafe and University), youth income (weekly) in AU\$ (no income, 1-39, 40-79, 80-119, 120-159, 160-199, 200-299, 300-399, 400-499, 500-599, 600-699, 700-799, 800-999, 1000-1499 or $\geq 1500$ ), marital status (never married, live together, married, separated-divorced-widowed) and living arrangements (living with $v$. not with family). Income was regrouped into three categories: low income (bottom 25\%); middle income (middle 50\%) and high income (top $25 \%$ ) based on relative distribution of average income ${ }^{(33)}$. Marital status and levels of education were aggregated into two and three categories, respectively. Also, information on vitamin supplement use (yes/no) was obtained by self-reports of young adults (Table 1 ).

\section{Assessment of dietary intake}

Usual dietary intake was assessed using the Cancer Council of Victoria's semiquantitaive and validated 101-food items $\mathrm{FFQ}^{(41,42)}$. Relative validity of nutrient intake for this FFQ has been documented, and the FFQ was found to be useful in the assessment of habitual intake in the Australian adult population $^{(42)}$. Correlation coefficients for energy-adjusted nutrient intake between $7-\mathrm{d}$ weighed food records and the FFQ ranged from 0.28 (vitamin A) to 0.78 (carbohydrate $)^{(42)}$. Study participants were asked to report on how often they usually consumed a specified food item (on a 10-point scale from 'never' to ' 3 or more times a day') over the previous 12 months, including six items on the consumption of alcoholic beverages. These were converted to daily equivalents for statistical analysis. The FFQ also included 10 short questions on the consumption 
Table 1 General characteristics among 2665 young adults (18-23 years) in Australia (2001-2004)

\begin{tabular}{|c|c|c|c|c|}
\hline & Total $(n$ 2665) & Men ( $n$ 1135) & Women ( $n$ 1530) & \\
\hline Characteristics* $^{*}$ & $\%$ & $\%$ & $\%$ & $P$-value \\
\hline \multicolumn{5}{|l|}{ Categorical variables } \\
\hline \multicolumn{5}{|l|}{ Smoking intensity (cigarettes/d) } \\
\hline Non-smokers & $66 \cdot 2$ & $63 \cdot 26$ & $68 \cdot 30$ & \multirow[t]{3}{*}{$<0.0001$} \\
\hline Smokers 1-19 & 29.2 & $30 \cdot 13$ & $28 \cdot 50$ & \\
\hline Smokers $\geq 20$ & $4 . \overline{7}$ & $6 \cdot 61$ & $3 \cdot 20$ & \\
\hline \multicolumn{5}{|c|}{ Alcohol consumption (standard drinks/d) } \\
\hline Non-consumers $(0)$ & $5 \cdot 9$ & $27 \cdot 58$ & $49 \cdot 08$ & \multirow[t]{4}{*}{$<0.0001$} \\
\hline Light consumers $(0.1-0.5)$ & 39.9 & $5 \cdot 29$ & 6.41 & \\
\hline Moderate consumers $(0.51-1)$ & $18 \cdot 9$ & $18 \cdot 68$ & $19 \cdot 02$ & \\
\hline Heavy consumers $(1.01-\geq 3.5)$ & $35 \cdot 3$ & $48 \cdot 46$ & $25 \cdot 49$ & \\
\hline \multicolumn{5}{|l|}{ Physical activity (sessions/week) } \\
\hline Sufficient $(\geq 5)$ & $33 \cdot 9$ & 33.04 & 34.44 & \multirow[t]{3}{*}{0.6808} \\
\hline Insufficient (1-4.99) & $56 \cdot 7$ & $57 \cdot 71$ & $56 \cdot 01$ & \\
\hline Sedentary $(0)$ & 9.4 & 9.25 & 9.54 & \\
\hline \multicolumn{5}{|l|}{ Time spent watching TV $(\mathrm{h} / \mathrm{d})$} \\
\hline$<2 \mathrm{~h}$ & $23 \cdot 8$ & $22 \cdot 56$ & $24 \cdot 71$ & \multirow[t]{2}{*}{$0 \cdot 1029$} \\
\hline $2-4 \mathrm{~h}$ & 43.4 & 42.47 & $44 \cdot 12$ & \\
\hline$\geq 4 \mathrm{~h}$ & $32 \cdot 8$ & 34.98 & $31 \cdot 18$ & \\
\hline \multicolumn{5}{|l|}{$\mathrm{BMI}\left(\mathrm{kg} / \mathrm{m}^{2}\right)$ category } \\
\hline Normal $(<25)$ & $67 \cdot 8$ & $65 \cdot 81$ & $69 \cdot 28$ & \multirow[t]{3}{*}{0.0124} \\
\hline Overweight (25-30) & $20 \cdot 7$ & 23.35 & 18.69 & \\
\hline Obese $(\geq 30)$ & 11.5 & $10 \cdot 84$ & $12 \cdot 03$ & \\
\hline \multicolumn{5}{|l|}{ Level of income (AUD/week) } \\
\hline High income (> 450) & $37 \cdot 1$ & $45 \cdot 99$ & $30 \cdot 52$ & \multirow[t]{3}{*}{$<0.0001$} \\
\hline Middle income (180-450) & 41.7 & 34.89 & $46 \cdot 67$ & \\
\hline Low income $(<180)$ & $21 \cdot 2$ & $19 \cdot 12$ & $22 \cdot 81$ & \\
\hline \multicolumn{5}{|l|}{ Level of education } \\
\hline Post-secondary school & $27 \cdot 5$ & $23 \cdot 88$ & $30 \cdot 13$ & \multirow[t]{3}{*}{$<0.0001$} \\
\hline Complete secondary school & $55 \cdot 1$ & 55.42 & 54.90 & \\
\hline Incomplete secondary school & 17.4 & $20 \cdot 70$ & 14.97 & \\
\hline \multicolumn{5}{|l|}{ Marital status } \\
\hline Not married† & $78 \cdot 9$ & 87.93 & $72 \cdot 22$ & \multirow[t]{2}{*}{$<0.0001$} \\
\hline Married or living together & $21 \cdot 1$ & $12 \cdot 07$ & $27 \cdot 78$ & \\
\hline \multicolumn{5}{|l|}{ Living arrangements } \\
\hline Living with parents & $60 \cdot 2$ & $69 \cdot 08$ & $53 \cdot 6$ & $<0.0001$ \\
\hline Not living with parents & 39.8 & 30.92 & $46 \cdot 4$ & \\
\hline Vitamin supplements use & & & & \\
\hline No & $67 \cdot 4$ & $76 \cdot 48$ & $60 \cdot 85$ & $<0.0001$ \\
\hline Yes & $32 \cdot 6$ & 23.52 & $39 \cdot 15$ & \\
\hline Continuous variables & & & & \\
\hline Age, years & & & & \\
\hline Mean & $20 \cdot 6$ & $20 \cdot 6$ & $20 \cdot 6$ & 0.5750 \\
\hline SD & 0.84 & 0.85 & 0.83 & \\
\hline Energy, kcal & & & & \\
\hline Mean & $2022 \cdot 1$ & 2473.3 & 1687.4 & $<0.0001$ \\
\hline SD & $766 \cdot 1$ & 751.5 & 583.7 & \\
\hline Fat, E \% & & & & \\
\hline Mean & $36 \cdot 8$ & $37 \cdot 8$ & $36 \cdot 1$ & $<0.0001$ \\
\hline SD & $5 \cdot 2$ & 4.8 & $5 \cdot 4$ & \\
\hline Carbohydrate, E \% & & & & \\
\hline Mean & $42 \cdot 0$ & 40.5 & $43 \cdot 2$ & $<0.0001$ \\
\hline SD & $6 \cdot 1$ & $5 \cdot 7$ & $6 \cdot 2$ & \\
\hline Protein, E \% & & & & \\
\hline Mean & $20 \cdot 0$ & $20 \cdot 1$ & $20 \cdot 0$ & 0.4157 \\
\hline SD & 3.0 & 2.9 & & \\
\hline
\end{tabular}

$\mathrm{E} \%$ is percent energy.

${ }^{*}$ Values are in \% or mean \pm SD.

†Not married includes never married and widowed, divorced and separated.

of fruit, vegetables, sugar, eggs, and the quantity and type of milk, cheese, bread and fat spreads. Photographs of different portion sizes of selected food items and dishes were included in the FFQ to assist in the calculation of daily energy and nutrient intake. These were estimated with the use of software developed by the Cancer Council of Victoria on the basis of Australian food composition table as contained in the Nutrient Data Table for Use in Australia (NUTTAB95), the national government food composition database of Australian foods ${ }^{(43)}$. 
Dietary patterns in young adults

Table 2 Food groupings used in dietary pattern analysis among 2665 young adults (18-23 years) in Australia (2001-2004)

\begin{tabular}{|c|c|}
\hline Food or food group $(\mathrm{g} / \mathrm{d})$ & Food items \\
\hline High-fat milk & Full cream milk; yoghurt, icecream, flavoured milk drink \\
\hline Low-fat milk & Reduced fat milk; skim milk \\
\hline Low-fat cheese & Low fat cheese, ricotta or cottage cheese \\
\hline High-fat cheese & Hard cheese; firm cheese; soft cheese; cream cheese \\
\hline Butter & Butter \\
\hline Spreads containing fat & Margarine; polyunsaturated margarine; monounsaturated margarine; butter and margarine blends \\
\hline Eggs & Boiled, fried, and scrambled eggs \\
\hline Poultry & Chicken with or without skin \\
\hline Red meat & Beef; veal; lamb; pork \\
\hline Processed meat & Sausages or frankfurters; hamburger; ham; bacon; corned beef, luncheon meats or salami \\
\hline Fish, other & Steamed, grilled or baked fish; tinned fish (salmon, tuna, sardines, etc.) \\
\hline Fish, fried & Fried fish (includes take away) \\
\hline Citrus fruit & Orange or other citrus fruit \\
\hline Other fruit & $\begin{array}{l}\text { Tinned or frozen fruit (any kind); strawberries; apples; pears; bananas; pineapple; watermelon, } \\
\text { rockmelon, honeydew etc.; apricots; peaches or nectarines; mango or paw paw; avocado }\end{array}$ \\
\hline Fruit juice & Fruit juice \\
\hline Vegetables & $\begin{array}{l}\text { Lettuce, endive, or other salad greens; Silverbeet or spinach; onion or leeks; beetroot; carrots, } \\
\text { pumpkin, peppers (capsicum); broccoli; cauliflower; cabbage or Brussel sprouts; cucumber; } \\
\text { celery; garlic; mushrooms; zucchini }\end{array}$ \\
\hline Tomatoes & Tomato sauce, tomato paste or dried tomatoes; fresh or tinned tomatoes; \\
\hline Potatoes & Roasted or fried (include hot chips), potatoes cooked without fat \\
\hline Legumes & $\begin{array}{l}\text { Bean sprouts; baked beans; soy beans, soy bean curd, tofu; other beans (chick peas, lentils etc.); } \\
\text { peas; green beans; soy milk }\end{array}$ \\
\hline Cereal products & Crackers, crispbreads, dry biscuits \\
\hline Other bread & High fibre white bread, refined white bread \\
\hline Wholemeal bread and whole grains & Wholemeal bread, rye bread, multigrain bread \\
\hline Breakfast cereals & $\begin{array}{l}\text { All bran, sultana bran, fibreplus, branflakes; weet bix, vita brits, weeties; cornflakes, nutrigrain, } \\
\text { special K; porridge; muesli }\end{array}$ \\
\hline Rice & Rice (all type) \\
\hline Pasta & Pasta or noodles (includes lasagne) \\
\hline Pizza & Pizza \\
\hline Meat pies and savoury pastries & Meat pies, pastries, quiche and other savoury pastries \\
\hline Snacks & Maize chips, potato crisps, twisties \\
\hline Chocolate & Chocolates \\
\hline Cakes and Pastries & Cakes, sweet pies, tarts, and other sweet pastries; sweet biscuits \\
\hline Sugar & Sugar \\
\hline Nuts & Nuts \\
\hline Non-fat spreads & Jam, marmalade, honey, syrup; Vegemite, Marmite, Promite; Peanut butter \\
\hline
\end{tabular}

\section{Derivation of dietary patterns}

To derive dietary patterns, first the 95 food items (excluding six items on alcohol intake) were grouped into 33 food groups on the basis of the similarity of food type, nutrient composition or culinary usage to reduce within person variation in dietary intake. Individual food items that constituted a distinct item (e.g. eggs, butter, pizza) or those thought to represent a particular eating pattern (e.g. potato, fruit juice, sugar) on their own were preserved (Table 2). We computed the average intake (in grams) of each participant's food group by summing the intake of the individual foods that made up each food group.

Dietary patterns were derived applying factor analysis with principal component extraction (PROC FACTOR in SAS) based on the consumption of 33 predefined food groups. The factor scores obtained were rotated by orthogonal transformation to achieve uncorrelated and more interpretable structures ${ }^{(44)}$. We considered solutions containing 2-4 factors. After examining factor solutions with eigenvalues $>1 \cdot 25$, we chose a two-factor solution based on the break point of scree plot and interpretability of the factors ${ }^{(44)}$. We identified two main dietary patterns and named them based on food groups with high loadings ${ }^{(45)}$. Food groups with absolute value of factor loadings $\geq 0.3$ were considered as meaningfully contributing to each dietary pattern ${ }^{(46)}$. Inter-item reliability for foods groups with high loadings in each dietary pattern was assessed by Cronbach's $\alpha$ coefficients. Factor scores for each dietary pattern were calculated by summing the products of intakes of food groups weighted by loading coefficients ${ }^{(46)}$ as have been presented in Table 3 .

Initially, we conducted dietary pattern analysis for men and women separately. The gender-specific dietary patterns identified were similar in relation to the number of factors identified and the foods that loaded highly. Therefore, the combined cohort of men and women was used for the present analysis. Because alcohol intake was included in the analyses as a lifestyle correlate of dietary patterns, we excluded items measuring alcohol intake as these contribute to derive dietary patterns.

\section{Statistical analyses}

Normality of the continuous variables including standardised scores for each dietary pattern and computed food 
Table 3 Factor loadings of the principal dietary patterns identified among 2665 young adults (18-23 years) in Australia (2001-2004)*

\begin{tabular}{|c|c|c|}
\hline Food/food group & $\begin{array}{c}\text { Western dietary } \\
\text { pattern }\end{array}$ & $\begin{array}{l}\text { Prudent dietary } \\
\text { pattern }\end{array}$ \\
\hline High-fat milk & 0.51 & \\
\hline Low-fat milk & -0.46 & 0.26 \\
\hline Low-fat cheese & -0.41 & \\
\hline High-fat cheese & 0.41 & \\
\hline Spreads containing fat & 0.33 & \\
\hline Eggs & 0.22 & 0.20 \\
\hline Poultry & 0.43 & 0.29 \\
\hline Red meat & 0.56 & 0.24 \\
\hline Processed meat & 0.70 & \\
\hline Fish, other & 0.19 & 0.44 \\
\hline Fish, fried & 0.52 & 0.22 \\
\hline Citrus fruit & & 0.32 \\
\hline Other fruit & & 0.53 \\
\hline Fruit juice & & 0.38 \\
\hline Vegetables & & 0.49 \\
\hline Tomatoes & & 0.33 \\
\hline Potatoes & & 0.30 \\
\hline Legumes & & 0.40 \\
\hline Cereal products & & 0.49 \\
\hline White bread & 0.52 & -0.32 \\
\hline Wholemeal bread & -0.42 & 0.40 \\
\hline Breakfast cereals & & 0.43 \\
\hline Rice, all type & & 0.47 \\
\hline Pasta or noodles & 0.25 & 0.47 \\
\hline Pizza & 0.56 & \\
\hline Meat pies and savory pastries & 0.55 & \\
\hline Snacks & 0.59 & \\
\hline Chocolate & 0.33 & 0.21 \\
\hline Cakes and pastries & 0.44 & 0.30 \\
\hline Sugar & 0.26 & \\
\hline Nuts & & 0.43 \\
\hline Non-fat spreads & 0.23 & 0.45 \\
\hline Explained variance (\%) & $14 \cdot 11$ & 9.23 \\
\hline Cronbach's $\alpha$ & 0.77 & 0.70 \\
\hline
\end{tabular}

*Absolute values $<0.20$ for each factor loadings were not shown for simplicity.

groups values $(\mathrm{g} / \mathrm{d})$ used to drive dietary patterns and age (in years) was checked visually using Q-Q plots of residuals against the predicted values, histograms and observing the skewness and kurtosis measures of their respective standard errors. Each of the dietary pattern scores and age were approximately normally distributed. However, computed food groups had positively skewed distributions; hence, their values were log-transformed before deriving dietary patterns. The standardised values of the two derived dietary pattern scores were considered as separate outcomes, and multivariable linear regression analysis was used for the main analyses. Differences in continuous and categorical variables between males and females were compared using the independent sample t-test and chi-square, respectively.

We first performed bivariate analyses to identify candidate variables for multivariable models using significance level of $<0 \cdot 20$. All explanatory variables had $P$-values $<0.20$ for the Western dietary pattern and hence, all of them were entered into a series of multivariable model. Despite having $P$-values $>0.20$ in bivariate analyses for a prudent dietary pattern, living arrangements and gender of participants were maintained in multivariable model based on previous findings ${ }^{(28,31,39)}$. Before fitting multivariable models for each dietary pattern, we examined multicollinearity among the explanatory lifestyle variables with the use of the variance inflation factor and Pearson correlation coefficients. Further, we examined evidence of interaction terms between gender and each lifestyle correlate examined. There was no evidence of collinearity or interaction observed in the current analysis. Thus, all analyses were based on the entire cohort of men and women.

To identify lifestyle variables significantly correlated with dietary patterns of young adults, a series of multivariable linear regression models were fitted separately for each dietary pattern adjusting for covariates including sociodemographic variables and energy intakes. The first two models were presented in Supplemental Table 1. In the first model, we run a model containing explanatory lifestyle variables including levels of smoking, alcohol consumption, physical activity, watching TV and BMI category. In the second model, we adjusted for covariates including age in years, gender, income, education, marital status, living arrangements and vitamin supplement use. In the fully adjusted model, we additionally adjusted for total energy intake of respondents (Table 4). All tests were two-sided and $P$-value $<0.05$ was considered statistically significant. The results are mean differences in dietary patterns scores across levels of examined lifestyle correlates and reported as standardised and unstandardised beta coefficients $(\beta \mathrm{s})$ with their $95 \%$ CIs presented in Table 4 and Supplemental Table 2, respectively. Also, standardised $\beta$ s are presented in Supplemental Table 2. All analyses were performed using SAS for window (version 9.4; SAS Institute).

As a sensitivity analysis, we fitted separate models to test if total effect of a lifestyle variable of interest out of four of these variables, namely physical activity, watching TV, smoking and alcohol consumption included in this study changes after successively leaving three or fewer others out of a fully adjusted model (Table 4) to have estimates for each from models with different covariate subsets. These sensitivity analyses did not substantially alter the estimates. Thus, we interpret total effects from a fully adjusted model above containing all lifestyle variables adjusted for covariates $^{(47)}$.

\section{Results}

\section{Characteristics of the study participants}

Data from a total of 2665 young adults was used for this analysis. Sociodemographic and lifestyle characteristics are presented in Table 1. Mean $\pm \mathrm{SD}$ age of participants was $20 \pm 0.8$ years (range $18-23$ years). A majority of the participants were females ( $n$ 1530; 57.3\%) and not married $(72.9 \%)$. A higher proportion of participants completed secondary school $(55.1 \%)$ and were in the middle-income 
Table 4 Standardised regression coefficients $(95 \% \mathrm{Cl})$ in fully adjusted models according lifestyle factors among 2665 young adults (18-23 years) in Australia (2001-2004)*

\begin{tabular}{|c|c|c|c|c|c|c|}
\hline \multirow[b]{2}{*}{ Characteristics } & \multicolumn{3}{|c|}{ Prudent pattern } & \multicolumn{3}{|c|}{ Western pattern } \\
\hline & $\beta$ & $95 \% \mathrm{Cl}$ & $P$-value & $\beta$ & $95 \% \mathrm{Cl}$ & $P$-value \\
\hline \multicolumn{7}{|l|}{ Physical activity (sessions/week) } \\
\hline Sufficient $(\geq 5)$ & Reference & & & Reference & & \\
\hline Insufficient (1-4) & -0.12 & $-0.15,-0.09$ & $<0.0001$ & 0.14 & $0.11,0.17$ & $<0.0001$ \\
\hline No physical activity (0) & -0.14 & $-0.17,-0.11$ & $<0.0001$ & $0 \cdot 11$ & $0.08,0.14$ & $<0.0001$ \\
\hline \multicolumn{7}{|l|}{ Time spent watching TV $(\mathrm{h} / \mathrm{d})$} \\
\hline$<2 \mathrm{~h}$ & Reference & & & Reference & & \\
\hline $2-4 \mathrm{~h}$ & -0.06 & $-0.09,-0.02$ & 0.0037 & 0.07 & $0.04,0.11$ & $<0.0001$ \\
\hline$\geq 4 \mathrm{~h}$ & -0.12 & $-0.16,-0.09$ & $<0.0001$ & 0.11 & $0.07,0.14$ & $<0.0001$ \\
\hline \multicolumn{7}{|l|}{ Smoking (cigarettes/d) } \\
\hline Non-smokers & Reference & & & Reference & & \\
\hline Smokers $1-19$ & -0.05 & $-0.09,-0.02$ & 0.0007 & 0.06 & $0.03,0.08$ & $<0.0001$ \\
\hline Smokers $\geq 20$ & -0.08 & $-0.12,-0.05$ & $<0.0001$ & 0.05 & $0.02,0.08$ & 0.0005 \\
\hline \multicolumn{7}{|c|}{ Alcohol consumption (standard drinks/d) } \\
\hline Non-consumers $(0)$ & -0.02 & $-0.05,0.01$ & 0.2902 & -0.003 & $-0.03,0.02$ & 0.8449 \\
\hline Light consumers $(0.1-0.5)$ & Reference & & & Reference & & \\
\hline Moderate consumers $(0.51-1)$ & 0.03 & $-0.01,0.06$ & 0.1251 & 0.01 & $-0.02,0.04$ & 0.5277 \\
\hline Heavy consumers $(1.01-\geq 3.5)$ & -0.03 & $-0.06,0.01$ & 0.1037 & 0.04 & $0.01,0.07$ & 0.0095 \\
\hline \multicolumn{7}{|l|}{$\mathrm{BMI}\left(\mathrm{kg} / \mathrm{m}^{2}\right)$ category } \\
\hline Normal $(<25)$ & Reference & & & Reference & & \\
\hline Overweight (25-30) & 0.04 & $0.01,0.07$ & 0.0163 & -0.04 & $-0.07,-0.02$ & 0.0014 \\
\hline Obese $(\geq 30)$ & 0.04 & $0.01,0.07$ & 0.0169 & -0.03 & $-0.06,-0.01$ & 0.0191 \\
\hline
\end{tabular}

*Models for each dietary pattern were run with the following lifestyle variables included: smoking, alcohol consumption, physical activity, time spent watching TV and BMI category. All results are adjusted for age in years, gender, income, education, marital status, living arrangement, vitamin supplement use and total energy intake.

(41.7\%) group. Two-thirds of participants were nonsmokers, while the majority of smokers smoked fewer than 20 cigarettes a day. A majority of the participants were light (39.9\%) and heavy (35.3\%) consumers of alcohol. A majority of the participants had insufficient ( $1-5$ sessions a week) physical activity and viewed TV for $2-4 \mathrm{~h} / \mathrm{d}$, respectively. Nearly two-thirds of participants were living with their parents and reported using no vitamin supplements, while nearly one-third were overweight or obese (BMI $\geq 25 \mathrm{~kg} / \mathrm{m}^{2}$ ).

Differences in sociodemographic and lifestyle characteristics are also presented by sex. Compared to males, female participants are more likely to be poor, not married, not living with parents, obese, non-users of vitamin supplements and less likely to be overweight and heavy smokers and alcohol consumers. Regarding macronutrient intakes, males had a higher total energy intake and higher percent energy from fat, while females had a higher percent energy from carbohydrate; there was no significant difference in energy intake from protein between males and females (Table 1).

\section{Characterising dietary patterns}

Using a total cohort of men and women, we identified two major dietary patterns, the Western and prudent patterns that collectively explained $21.6 \%(13.0 \%$ and $8.6 \%$, respectively) of variation in dietary intakes in young adults. The Western pattern was characterised by high consumption of red meats, processed meat, poultry, fried fish, pizza, meat pies and savoury pastries, high-fat dairy products, fat containing spreads, white bread, pasta, snacks, chocolate and cakes and pastries and low consumption of low-fat dairy products and wholemeal bread. The prudent pattern was characterised by high consumption of vegetables, fruit, fish, potatoes, legumes, cereals, wholemeal bread, rice, pasta, nuts and non-fat containing spreads and low consumption of white bread. Inter-item reliability in each dietary pattern was assessed by Cronbach's $\alpha$ coefficients and these were 0.74 for each pattern. Factor loadings for each dietary pattern are presented in Table 3 . The standardised factor scores for the Western dietary pattern ranged from -3.46 to 2.56 and was from -3.21 to 3.11 for a prudent pattern. The lower and higher dietary pattern scores suggest lesser and greater adherence to a particular dietary pattern.

\section{Lifestyle correlates of dietary patterns}

Table 4 presents lifestyle correlates of each dietary pattern fully adjusted for age, gender, income, education, marital status, living arrangements, vitamin supplement and total energy intakes. The strongest lifestyle correlates of both a prudent and the Western dietary patterns are measures of physical activity, time spent watching TV and smoking in each fully adjusted model. Participants with a greater adherence to a prudent dietary pattern are less likely to engage in lower levels of physical activity, are less likely to watch TV for extended periods of time and are less likely to be light and heavy smokers. There was also a weaker but significant positive association of a prudent dietary pattern with BMI of young adults; those with a greater adherence to 
this pattern are more likely to be overweight and obese. Alcohol consumption was not associated with a prudent dietary pattern.

For each lifestyle variable correlated with a prudent dietary pattern, a similar strength but opposite direction of association with the Western dietary pattern is observed. Young adults who adhere more to the Western dietary pattern are more likely to engage in lower levels of physical activity, are more likely to watch TV for extended periods of time and are more likely to be light and heavy smokers. Alcohol consumption and BMI were weakly associated with the Western dietary pattern. Those with a greater adherence to the Western pattern are more likely to be heavy consumers of alcohol and are less likely to be overweight and obese (Table 4).

We also evaluated the associations between dietary pattern scores and energy and energy adjusted nutrient intakes of participants. The two dietary patterns differ with the Western pattern being positively and a prudent pattern being negatively associated with percent energy from fat and intakes of total fat, saturated fat, monounsaturated fat, total cholesterol and sodium. By contrast, the Western pattern is negatively and a prudent pattern is positively associated with percent energy from carbohydrate and protein and intakes of protein, carbohydrate, dietary fibre, sugar, starch, total beta-carotene and selected vitamins and minerals (see online Supplemental Table 3).

\section{Discussion}

We have identified two main dietary patterns, the Western and prudent patterns in this large study of young adults aged 18-23 years. The Western pattern is correlated with higher consumption of processed and red meat, poultry, high-fat dairy products, refined grains, takeaway foods, fried fish, fat and non-fat spreads and snacks and sweets, whereas a prudent pattern is correlated with higher consumption of fruit, vegetables, potatoes, legumes, nuts, whole grains, fish, cereals and non-fat spreads. The two dietary patterns explained $21.6 \%$ of the variance in dietary intake in this study. This is consistent with findings from studies involving young adults from Brazil and the $\mathrm{UK}^{(21,23)}$. Studies of young adults in the USA and in Canada have reported higher (31\%) and lower (15.6\%) total variance in food intakes explained in the dietary patterns that they have identified ${ }^{(19,25)}$.

We labeled the dietary patterns identified in this study based on previous literature ${ }^{(45)}$. However, these dietary patterns are slightly different from those reported in other studies because food group 'cakes and pastries' is correlated with both patterns in this study. Multiple loadings of food items have been observed in previous studies ${ }^{(22)}$.

Different studies report varying numbers and types of dietary patterns in nutritional epidemiology. Consistent with our results, three studies of young adults have identified similar types of dietary patterns to those reported in the current study ${ }^{(19,22,24)}$. One study among US young adults has identified Western and prudent dietary patterns $^{(19)}$. A study from Denmark has identified traditional Western and green dietary patterns in young adults ${ }^{(22)}$, with comparable food composition to the Western pattern and prudent pattern in this study. Finally, a study of young women in the UK has identified the prudent and highenergy dietary patterns, the latter pattern having similar characteristics to the Western pattern observed in this study $^{(24)}$. Moreover, studies involving adult women from the "Mamma \& Bambino" cohort in Italy have identified the Western and prudent dietary patterns with similar food composition to those observed in this study ${ }^{(48,49)}$. However, studies in young adult populations in Brazil and UK have identified up to five dietary patterns with varying food compositions $^{(20,21,23)}$. A study involving young (25-30 years) and middle-aged (50-55 years) women in Australia has identified up to six dietary patterns composed in various ways $^{(37)}$. The variations in types of dietary patterns identified using data-driven methods (e.g. principal components analysis) may reflect variations of foods included in the specific studies, the distribution of these foods in the data set and inconsistencies among the studies in defining and interpreting dietary patterns ${ }^{(50,51)}$. The variations in dietary patterns among studies using data-driven methods to define dietary patterns could arise from differences in methods used to assess dietary intake as well as differences in food grouping and the number of food items and food groups used in the statistical analysis ${ }^{(52)}$. To address these inconsistencies, researchers have suggested looking at the results of individual studies to ascertain which foods have substantially contributed to the dietary patterns of interest $^{(50,51)}$.

The current study suggests that dietary patterns are associated with the broader lifestyles of young adults. Lower levels of physical activity, extended duration of watching TV and smoking were the strongest correlates of each dietary pattern. In this study, participants with lower levels of physical activity had less adherence to a prudent dietary pattern, while they had greater adherence to the Western pattern. Other studies have also reported that more active participants had higher scores for healthier dietary patterns and lower scores for less healthy dietary patterns in young men and women in Brazil and the USA, as well as in a study of young and middle-aged women in Australia ${ }^{(19,20,37)}$. However, the study in the USA found no association between physical activity and a prudent dietary pattern with this latter null finding in the USA study possibly explained by a relatively larger sample size in this study ${ }^{(19)}$. We have found that participants with longer duration of watching TV had lower adherence to a prudent dietary pattern and a higher adherence to the Western pattern. A few studies involving young women in the UK and university students in the USA have reported no association between duration of watching TV and any of the identified dietary 
patterns $^{(18,24)}$. This could be explained by the fact that both studies had relatively small samples and may have been underpowered to detect a difference. However, a study in Brazilian adolescents found a positive association between eating while watching TV and an unhealthy dietary pattern, partly supporting the findings observed in the current study ${ }^{(53)}$. Results of this study suggest young adults with lower levels of physical activity and longer duration of watching TV are less likely to consume healthy foods rich in micronutrients and fiber, and they are more likely to consume unhealthy foods rich in fat and dietary cholesterol as has been reported in other studies ${ }^{(7,16,17)}$.

In this study, smokers were less likely to adhere to a prudent dietary pattern and more likely to adhere to the Western pattern. Our findings are consistent with previous studies of young adults in Brazil and in the USA, as well as with a study of young and middle-aged women in Australia ${ }^{(19,20,37)}$. Evidence from previous studies of young adults shows that smoking has been associated with reduced intakes of fruits, essential fatty acids, fiber and micronutrients and higher intakes of energy, total fat, saturated fat and dietary cholesterol ${ }^{(14,15)}$.

In this study, heavy consumption of alcohol was positively associated with the Western dietary pattern, whereas it showed no association with a prudent pattern. Our findings in part are consistent with findings of a study involving a pooled cohort of young and middle-aged women in Australia ${ }^{(37)}$. Higher consumption of alcohol has been associated with lower consumption of healthy foods and higher consumption of unhealthy foods among Spanish university students ${ }^{(13)}$. A study in the US young adults found no association between alcohol consumption, and the Western and prudent dietary patterns ${ }^{(19)}$, partly supporting our findings. The observed different results between the US study and the current study might reflect the relatively lower study power in the US study.

Unexpectedly, we observed that obese and overweight participants had respective higher and lower scores for the prudent and the Western dietary patterns. Studies of young women and university students in the UK observed no association between BMI and any of the identified dietary patterns ${ }^{(23,24)}$. These studies in the UK had small samples, possibly explaining the observed differences ${ }^{(23,24)}$. Our findings are consistent with a study involving young and middle-aged women in Australia ${ }^{(37)}$. The results of this study could suggest that people may select healthy foods when they become obese or overweight $^{(38)}$. Also, underreporting and omitting snacks are common problems in accurately assessing dietary intakes of general population, and more so in obese and overweight people, partly explaining the current findings $(54,55)$.

Notably, a number of lifestyle variables in the fully adjusted model are correlated and it is arguable which of these effects precedes the other in this cross-sectional study. For example, physical activity could arguably reduce time spent watching TV or watching TV could, arguably, reduce the time available for physical activity. It could be suggested that some lifestyle variables in the fully adjusted model could be treated as moderators in assessing their associations with dietary patterns. These considerations while most relevant in longitudinal study designs are not central to our argument. Our aim has been to establish whether dietary patterns are associated with and hence, part of the more general aspect of the lifestyles of respondents. Furthermore, in sensitivity analyses, removing lifestyle variables which are arguably moderators from fully adjusted models (e.g. physical activity in the association between time spent watching TV and dietary patterns or vice versa) has no observable impact on the broader associations we have observed.

\section{Strengths and limitations of the study}

Our study has several strengths. We used data from a community-based MUSP birth cohort and we had a relatively large sample size included in the analyses. Also, we used dietary data from a validated FFQ that has been designed for use in adult population in Australia ${ }^{(42)}$. Observing the clustering of dietary and other habits of lifestyle in young adults is another strength of our study, given young adulthood is arguably a period in the life course during which a range of lifestyle may develop including habits of dietary intake, physical activity, sedentary activity, cigarrete smoking and alcohol consumption. Furthermore, this study, to our knowledge, is the first to use data-driven method to assess dietary patterns of both young men and women in Australia.

Some limitations of this study are acknowledged. First, our study used a cross-sectional data from the MUSP cohort during the 21-year follow-up (2001-2004) and hence, causality in the observed associations cannot be ascertained. The narrow age range (18-23 years) of respondents in our sample is acknowledged. We have noted that some data was excluded due to some missing food items in the FFQ and implausible energy intake which might have affected some estimates in our study.

We have used data that have been collected during 2001-2004 and our findings might not reflect dietary pattern of the current generation. There have been declines in the availability and intake of added or refined sugars in adult men (such change in women was NS) and sugarsweetened beverages in men and women adults over the period of 1980/1995-2011 in Australia ${ }^{(56,57)}$. Also, there has been improved consumption of healthy foods such as fruit, vegetables, nuts and whole grain cereals during 1995-2011 in Australian adults ${ }^{(57)}$. However, more recent data indicate the majority of adult Australians, particularly in the younger age group, are failing to meet national dietary guidelines. Only $24 \%$ of women and $15 \%$ of men have dietary intakes of both the fruit and vegetable consistent with national guidelines ${ }^{(58)}$. 
There has been substantial loss to follow-up in the MUSP cohort, a long-term follow-up study of mothers and their offspring for more than 30 years. Those disproportionately lost to follow-up are more likely to involve respondents who are young and socially disadvantaged, of separated or divorced marital status and have higher rates of unhealthy lifestyles ${ }^{(30,31)}$. Loss to follow-up affects sample means, but they rarely affect estimates of association ${ }^{(59,60)}$. Similar estimates of association in the groups retained in the study and those lost to follow-up are reported in a study that has evaluated the impact of biased loss to follow-up using the MUSP birth cohort data ${ }^{(60)}$. The MUSP birth cohort is not representative of the entire Australian population, but the cohort comprises a broad cross-section of the population $^{(31)}$.

\section{Conclusion}

Our study has identified two main dietary patterns among young adults, namely the Western and prudent patterns. The lifestyles of young adults including physical activity, watching TV, smoking and alcohol consumption are associated with these dietary patterns. These findings suggest that there is clustering of lifestyle features associated with the main patterns of food consumption observed in a developed economy like Australia. This clustering involves a number of lifestyle features such as physical activity, sedentary activity, cigarette smoking and alcohol consumption. This clustering of factors raises three important issues. Firstly, from a research perspective any association between patterns of food consumption and health outcomes needs to take account of this clustering. Secondly, from a clinical or therapeutic perspective, efforts to modify diet in young adults need to include a focus on the multiple lifestyles of those whose patterns of food consumption predisposes them to a range of adverse health outcomes. Thirdly, from policy perspective, because a range of lifestyles are partly formed during young adulthood, nutrition and health policies should target emerging adults to promote healthy lifestyle and subsequently reduce the risk of unhealthy lifestyle related adverse health outcomes at later ages. Finally, evaluating the contributing factors to the development of unhealthy lifestyles and their clustering over time needs to be determined using prospective cohort studies.

\section{Acknowledgements}

Acknowledgements: The authors would like to acknowledge Greg Shuttlewood, a MUSP data manager, for his assistance with providing important variables in the MUSP data to create analytical cohort for the current study. Financial support: Funding for the MUSP study was received from National and Medical Research Council (NHMRC) (grant number NHMRC Grant number 210298). None of the authors have received commercial or private funding for any component to this particular study. The funder, NHMRC, had no role in the design, analysis or writing of this article. Conflict of interest: The authors declare that they have no competing interests. Authorship: T.W.U. is supported by the UQ Research Training Scholarship. T.W.U.: conceptualised the study and performed the statistical analysis, drafted the paper mainly under J.M.N. supervision, and had primary responsibility for the final content; all authors critically reviewed drafts of the paper and provided advice for the final version; P.H.L.: specifically provided important intellectual content on measures of dietary patterns and the whole manuscript. Etbics of human subject participation: MUSP birth cohort was conducted according to the guidelines laid down in the Declaration of Helsinki. In this cohort, written informed consent from the mothers was obtained during all data collection phases of the study, and at 21 years, informed consent was obtained from the offspring. Ethics committees at the Mater Hospital and the University of Queensland approved each phase of the study.

\section{Supplementary material}

For supplementary material accompanying this paper visit https://doi.org/10.1017/S1368980021003864

\section{References}

1. Schoenborn CA (1986) Health habits of U.S. adults, 1985: the "Alameda 7" revisited. Public Health Rep 101, 571-580.

2. Arnett JJ (2000) Emerging adulthood. A theory of development from the late teens through the twenties. Am Psychol 55, 469-480.

3. Schüz B (2017) Socio-economic status and theories of health behaviour: time to upgrade a control variable. Br J Health Psychol 22, 1-7.

4. Brug J, Kremers SP, Lenthe FV et al. (2008) Environmental determinants of healthy eating: in need of theory and evidence: symposium on 'behavioural nutrition and energy balance in the young'. Proc Nutr Soc 67, 307-316.

5. Arnett JJ, Žukauskienè R \& Sugimura K (2014) The new life stage of emerging adulthood at ages 18-29 years: implications for mental health. Lancet Psychiatry 1, 569-576.

6. Allman-Farinelli M, Partridge SR \& Roy R (2016) Weightrelated dietary behaviors in young adults. Curr Obes Rep $\mathbf{5}, 23-29$.

7. Laska MN, Hearst MO, Lust K et al. (2015) How we eat what we eat: identifying meal routines and practices most strongly associated with healthy and unhealthy dietary factors among young adults. Public Health Nutr 18, 2135-2145.

8. Hobbs M, Duncan MJ, Collins P et al. (2018) Clusters of health behaviours in Queensland adults are associated with different socio-demographic characteristics. J Public Health 41, 268-277.

9. Plaza-Díaz J, Molina-Montes E, Soto-Méndez MJ et al. (2020) Clustering of dietary patterns and lifestyles among Spanish children in the EsNuPI study. Nutrients 12, 2536. 
10. Movassagh EZ, Baxter-Jones ADG, Kontulainen $\mathrm{S}$ et al. (2017) Tracking dietary patterns over 20 years from childhood through adolescence into young adulthood: the Saskatchewan pediatric bone mineral accrual study. Nutrients 9, 990.

11. Biddle SJH, Pearson N, Ross GM et al. (2010) Tracking of sedentary behaviours of young people: a systematic review. Prev Med 51, 345-351.

12. Fergusson DM, Lynskey MT \& Horwood LJ (1995) The role of peer affiliations, social, family and individual factors in continuities in cigarette smoking between childhood and adolescence. Addiction 90, 647-659.

13. Scholz A, Navarrete-Muñoz EM, Garcia de la Hera M et al. (2016) Alcohol consumption and Mediterranean diet adherence among health science students in Spain: the DiSA-UMH study. Gac Sanit 30, 126-132.

14. Steptoe A, Wardle J, Cui W et al. (2002) Trends in smoking, diet, physical exercise, and attitudes toward health in European university students from 13 countries, 1990-2000. Prev Med 35, 97-104.

15. Dallongeville J, Marécaux N, Fruchart J-C et al. (1998) Cigarette smoking is associated with unhealthy patterns of nutrient intake: a meta-analysis. J Nutr 128, 1450-1457.

16. Gillman MW, Pinto BM, Tennstedt S et al. (2001) Relationships of physical activity with dietary behaviors among adults. Prev Med 32, 295-301.

17. Thomson M, Spence JC, Raine K et al. (2008) The association of television viewing with snacking behavior and body weight of young adults. Am J Health Promot 22, 329-335.

18. Mueller MP, Blondin SA, Korn AR et al. (2018) Behavioral correlates of empirically-derived dietary patterns among University students. Nutrients 10, 716.

19. Deshmukh-Taskar PR, O'Neil CE, Nicklas TA et al. (2009) Dietary patterns associated with metabolic syndrome, sociodemographic and lifestyle factors in young adults: the Bogalusa heart study. Public Health Nutr 12, 2493-2503.

20. Olinto MTA, Willett WC, Gigante DP et al. (2011) Sociodemographic and lifestyle characteristics in relation to dietary patterns among young Brazilian adults. Public Health Nutr 14, 150-159.

21. Arruda SPM, da Silva AAM, Kac G et al. (2014) Socioeconomic and demographic factors are associated with dietary patterns in a cohort of young Brazilian adults. BMC Public Health 14, 654.

22. Hare-Bruun H, Togo P, Andersen LB et al. (2011) Adult food intake patterns are related to adult and childhood socioeconomic status. J Nutr 141, 928-934.

23. Sprake EF, Russell JM, Cecil JE et al. (2018) Dietary patterns of university students in the UK: a cross-sectional study. Nutr J 17, 90.

24. Borland SE, Robinson SM, Crozier SR et al. (2008) Stability of dietary patterns in young women over a 2 -year period. Eur $J$ Clin Nutr 62, 119-126.

25. Brenner DR, Boucher BA, Kreiger N et al. (2011) Dietary patterns in an ethnoculturally diverse population of young Canadian adults. Can J Diet Pract Res 72, e161-e168.

26. VanKim NA, Larson N \& Laska MN (2012) Emerging adulthood: a critical age for preventing excess weight gain? Adolesc Med State Art Rev 23, 571-588.

27. Nelson MC, Story M, Larson NI et al. (2008) Emerging adulthood and college-aged youth: an overlooked age for weight-related behavior change. Obesity 16, 2205-2211.

28. Wardle J, Haase AM, Steptoe A et al. (2004) Gender differences in food choice: the contribution of health beliefs and dieting. Ann Behav Med 27, 107-116.

29. Munt AE, Partridge SR \& Allman-Farinelli M (2017) The barriers and enablers of healthy eating among young adults: a missing piece of the obesity puzzle: a scoping review. Obes Rev 18, 1-17.

30. Najman JM, Alati R, Bor W et al. (2015) Cohort Profile update: the Mater-University of Queensland study of pregnancy (MUSP). Int J Epidemiol 44, $78 \mathrm{f}$.

31. Lahmann PH, Williams GM, Najman JM et al. (2017) Mother-adult offspring resemblance in dietary intake: a community-based cohort study in Australia. Am J Clin Nutr 105, 185-193.

32. Booth M, Owen N, Banman A et al. (1995) Active and Inactive Australians: Assessing and Understanding Levels of Physical Activity: Australian Fitness Survey 1990-1991. Canberra, ACT: Department of the Environment, Sport and Territories, Commonwealth of Australia.

33. Berlin JA, Longnecker MP \& Greenland S (1993) Meta-analysis of epidemiologic dose-response data. Epidemiology 4, 218-228.

34. Australian Institute of Health Welfare (2003) The Active Australia Survey: A Guide and Manual for Implementation, Analysis and Reporting. Canberra: AIHW.

35. World Health Organization (2000) Obesity: Preventing and Managing the Global Epidemic. Geneva, Switzerland: World Health Organization.

36. Waters A-M (1993) Assessment of Self-Reported Height and Weight and Their Use in the Determination of Body Mass Index: Analysis of Data from the 1989 Risk Factor Prevalence Survey. Canberra, Australia: Australian Institute of Health and Welfare.

37. Mishra GD, McNaughton SA, Ball K et al. (2010) Major dietary patterns of young and middle aged women: results from a prospective Australian cohort study. Eur J Clin Nutr 64, 1125-1133

38. Sanchez-Villegas A, Delgado-Rodriguez M, MartinezGonzalez MA et al. (2003) Gender, age, socio-demographic and lifestyle factors associated with major dietary patterns in the Spanish project SUN (Seguimiento Universidad de Navarra). Eur J Clin Nutr 57, 285-292.

39. Elstgeest LEM, Mishra GD \& Dobson AJ (2012) Transitions in living arrangements are associated with changes in dietary patterns in young women. J Nutr 142, 1561-1567.

40. Dickinson A \& MacKay D (2014) Health habits and other characteristics of dietary supplement users: a review. Nutr $J \mathbf{1 3}, 14$

41. Ireland P, Jolley D, Giles G et al. (1994) Development of the Melbourne FFQ: a food frequency questionnaire for use in an Australian prospective study involving an ethnically diverse cohort. Asia Pac J Clin Nutr 3, 19-31.

42. Hodge A, Patterson AJ, Brown WJ et al. (2000) The anticancer council of Victoria FFQ: relative validity of nutrient intakes compared with weighed food records in young to middle-aged women in a study of iron supplementation. Aust N Z J Public Health 24, 576-583.

43. Lewis J, Milligan GC, Hunt A et al. (1995) NUTTAB 95: Nutrient Data Table for Use in Australia. Canberra: Commonwealth of Australia.

44. O'Rourke N \& Hatcher L (2013) A Step-By-Step Approach to Using SAS for Factor Analysis and Structural Equation Modeling. Cary, NC: SAS Institute.

45. Hu FB (2002) Dietary pattern analysis: a new direction in nutritional epidemiology. Curr Opin Lipidol 13, 3-9.

46. Kline P (1994) An Easy Guide to Factor Analysis. London, NY: Routledge.

47. Westreich D \& Greenland S (2013) The table 2 fallacy: presenting and interpreting confounder and modifier coefficients. Am J Epidemiol 177, 292-298.

48. Maugeri A, Barchitta M, Favara G et al. (2019) Maternal dietary patterns are associated with pre-pregnancy body 
mass index and gestational weight gain: results from the "Mamma \& Bambino" cohort. Nutrients 11, 1308.

49. Barchitta M, Maugeri A, Magnano San Lio R et al. (2019) Dietary patterns are associated with leukocyte LINE-1 methylation in women: a cross-sectional study in Southern Italy. Nutrients 11, 1843.

50. Schulze MB, Martínez-González MA, Fung TT et al. (2018) Food based dietary patterns and chronic disease prevention. BMJ 361, k2396.

51. Slattery ML, Boucher KM, Caan BJ et al. (1998) Eating patterns and risk of colon cancer. Am J Epidemiol 148, $4-16$.

52. Newby PK, Muller D \& Tucker KL (2004) Associations of empirically derived eating patterns with plasma lipid biomarkers: a comparison of factor and cluster analysis methods. Am J Clin Nutr 80, 759-767.

53. Maia EG, Silva L, Santos MAS et al. (2018) Dietary patterns, sociodemographic and behavioral characteristics among Brazilian adolescents. Rev Brasil Epidemiol 21, e180009.

54. Heitmann BL, Lissner L \& Osler M (2000) Do we eat less fat, or just report so? Int J Obes 24, 435-442.
55. Heitmann BL \& Lissner L (1995) Dietary underreporting by obese individuals - is it specific or non-specific? BMJ 311, 986-989.

56. Brand-Miller JC \& Barclay AW (2017) Declining consumption of added sugars and sugar-sweetened beverages in Australia: a challenge for obesity prevention. Am J Clin Nutr 105, 854-863.

57. Ridoutt B, Baird D, Bastiaans K et al. (2016) Changes in food intake in Australia: comparing the 1995 and 2011 national nutrition survey results disaggregated into basic foods. Foods 5, 40.

58. Hendrie G \& Noakes M (2017) Fruit, Vegetables and Diet Score: UCL. https://www.totalwellbeingdiet.com/media/ 2129/2017-csiro-fruit-vegetables-and-diet-score.pdf (accessed November 2020).

59. Howe LD, Tilling K, Galobardes B et al. (2013) Loss to follow-up in cohort studies: bias in estimates of socioeconomic inequalities. Epidemiology 24, 1-9.

60. Saiepour N, Najman JM, Ware R et al. (2019) Does attrition affect estimates of association: a longitudinal study. J Psychiatr Res 110, 127-142. 